\title{
Tumores metastáticos para os maxilares: uma revisão integrativa
}

\section{Metastatic tumors to the jaws: an integrative review}

\author{
José Wittor de Macêdo Santos ${ }^{1}$, Manuel Antonio Gordón-Núñez ${ }^{1}$ \\ 1. Faculdade de Odontologia da Universidade Estadual da Paraíba (UEPB), Araruna, PB, Brasil.
}

\begin{abstract}
Resumo
Introdução: Tumores metastáticos na região oral e perioral são extremamente raros. Objetivo: realizar uma revisão integrativa dos casos de metástases nos maxilares e sua relação com os tumores primários, os tipos histológicos e as principais localizações topográficas de ambos. Metodologia: Foi realizada uma pesquisa nas bases de dados MEDLINE, Google acadêmico e LILACS utilizando os seguintes critérios: artigos publicados na íntegra, no período de 2000 a 2016, em inglês, português e espanhol, case report e classical articles sobre o tema, utilizando os seguintes descritores: maxilares, mandíbula, maxila, metástases, incidência e seus mesh terms. Resultados: Foram incluídos um total de 35 artigos que cumpriam com todos os critérios. Foram ao todo 452 pacientes com metástases, dos quais $53.1 \%$ eram homens. A idade dos pacientes variou dos 4 meses a 90 anos. A mandíbula foi acometida em $55.7 \%$ dos casos, e a principal localização primária foi a mama, seguida pelo pulmão com 90 e 87 casos, respectivamente. Em 39\% da amostra, os tumores primários eram desconhecidos. 0 adenocarcinoma foi o principal padrão histopatológico sendo encontrado em $30.3 \%$ da amostra. Conclusão: 0 s tumores metastáticos dos maxilares estão relacionados com um diagnóstico desafiador e tardio com prognóstico sombrio de curta sobrevida. Acometem mais comumente a região posterior mandibular entre a quinta e oitava décadas de vida, em ambos os sexos. 0 cirurgião dentista tem grande papel no diagnóstico precoce destas lesões.
\end{abstract}

Palavras-chave: Câncer. Metástase. Maxilares. Cavidade oral. Revisão Integrativa.

\begin{abstract}
Introduction: Metastatic tumors in the oral and perioral region are extremely rare. Objective: To aimed at performing an integrative review of the cases of metastases in the jaws and their relation to primary tumors, histological types and the topographic locations of both. Methods: A search was carried out in the MEDLINE, Google academic and LILACS databases using the following criteria: full articles published, from 2000 to 2016 , in English, Portuguese and Spanish, case report and classical articles about the topic, using the following descriptors: jaws, mandible, maxilla, metastasis, incidence and their mesh terms. Results: 35 articles that fulfilled all the criteria were included. There were 452 patients with metastases, of which $53.1 \%$ were men. Patient's ages ranged from 4 months to 90 years. The mandible was affected in $55.7 \%$ of the cases, and the main primary sites were the breast and lung with 90 and 87 cases each, respectively. In $39 \%$ of the sample, the primary tumors were unknown. Adenocarcinoma was the main histopathological pattern found in $30.3 \%$ of the sample. Conclusion: Metastatic tumors of the jaws are related to a challenging and late diagnosis with gloomy prognosis of short survival. They most commonly affect the posterior mandibular region between the fifth and eighth decades of life, in both sexes. The dentist practitioner has a great role in the early diagnosis of these lesions.
\end{abstract}

Key words: Cancer. Metastasis. Jaws. Oral cavity. Integrative review.

\section{INTRODUÇÃO}

O câncer é uma doença complexa constituída por diversos processos patofisiológicos que incluem a divisão celular, perda da apoptose, migração celular e invasão de tecidos circunvizinhos e a distância. Lesões metastáticas que acompanham os maxilares são lesões extremamente raras, podendo ocorrer tanto em tecidos duros quanto em tecidos moles ${ }^{1,2}$. Apesar de qualquer tumor maligno ter a capacidade de metastatizar para a região maxilofacial, aparentemente há alguns locais de tumores primários com esta tendência, tais como os de pulmões, mama, rim, tireoide e próstata ${ }^{1-3}$. Em muitos casos, a metástase é o primeiro sinal da doença, com o tumor primário desconhecido até o diagnóstico da metástase oral ${ }^{4}$.

Segundo Sánchez-Jiménez et $\mathrm{al}^{4}$., os ossos gnáticos são mais atingidos por metástases que os tecidos moles da região oral e perioral, perfazendo aproximadamente $85 \%$ dos casos, destes, a mandíbula é a localização da lesão em $60 \%$ a $90 \%$ de todos os casos, sendo a região posterior a mais afetada. As metástases são relacionadas a um prognóstico sombrio por representarem frequentemente um estágio avançado da doença. Clinicamente estas se apresentam como lesões que podem mimetizar alterações de origem odontogênica, lesões hiperplásicas e/ou com aparência de lesões reativas como granuloma piogênico, epúlide fissurada, abscessos periodontais ou infecções locais na cavidade oral, com dor, inchaço e parestesia, dificultando seu diagnóstico e tornando-o desafiador ${ }^{3-5}$.

Este trabalho teve como objetivo realizar uma revisão integrativa da literatura analisando retrospectivamente os relatos de casos e pesquisas sobre as localizações de tumores 
secundários mais prevalentes em pacientes acometidos com tumores metastáticos nos maxilares publicados nos últimos anos, avaliando sua relação com os tumores primários, os tipos histológicos e as principais características clínicas e radiográficas.

\section{MÉTODOS}

\section{Pergunta de pesquisa}

Esta revisão foi conduzida inicialmente baseada no desenvolvimento da seguinte pergunta de pesquisa: Quais as localizações topográficas mais prevalentes de tumores primários e secundários, além do tipo histológico em pacientes acometidos com metástases nos maxilares?

\section{Estratégia de Busca}

Três bases de dados da Internet foram utilizadas para identificar documentos que satisfizessem o objetivo do estudo: a National Library of Medicine, Washington, DC (MEDLINE-PubMed), o Google Acadêmico e a base de Literatura Latino-Americana e do Caribe em Ciências da Saúde (LILACS). As bases de dados foram pesquisadas para estudos realizados até Janeiro de 2017 (Figura 1).

Figura 1. Pesquisa e seleção dos resultados

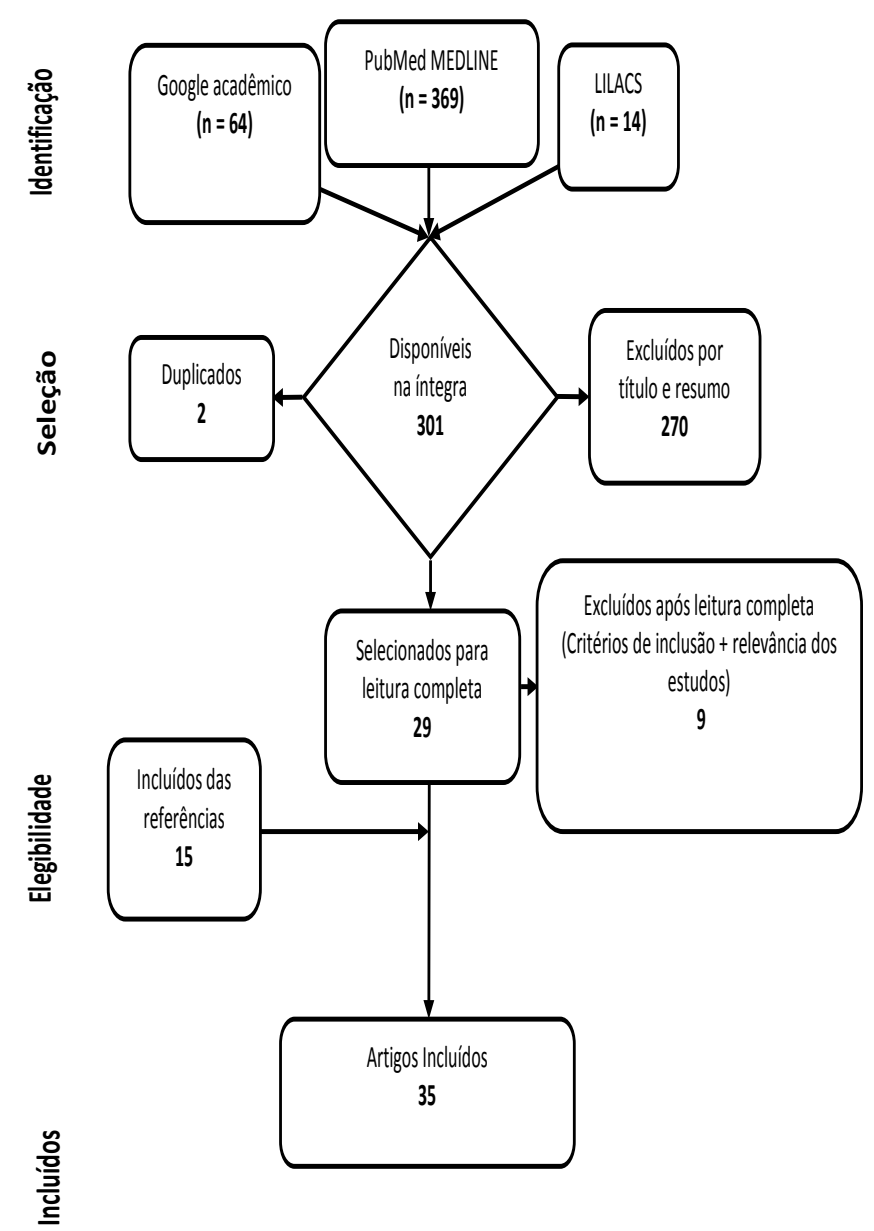

A estratégia de busca foi personalizada de acordo com a base de dados pesquisada. Os seguintes termos baseados na pergunta de pesquisa e na estratégia PICO foram utilizados na estratégia de pesquisa na MEDLINE-PubMed:

Population: Jaw OR Jaws OR Mandible OR Mandibles OR Mylohyoid Ridge OR Mylohyoid Ridges OR Ridge, Mylohyoid OR Ridges, Mylohyoid OR Mylohyoid Groove OR Groove, Mylohyoid OR Grooves, Mylohyoid OR Mylohyoid Grooves OR Maxilla OR Maxillas OR Maxillary Bone OR Bone, Maxillary OR Bones, Maxillary OR Maxillary Bones OR Maxillae

AND

Intervention: Neoplasm Metastasis OR Metastases, Neoplasm OR Neoplasm Metastases OR Metastasis OR Metastases OR Metastasis, Neoplasm OR Neoplasms, Unknown Primary OR Occult Primary Neoplasms OR Neoplasms, Occult Primary OR Neoplasm, Occult Primary OR Occult Primary Neoplasm OR Primary Neoplasm, Occult OR Primary Neoplasms, Occult OR Unknown Primary Neoplasms OR Neoplasm, Unknown Primary OR Primary Neoplasm, Unknown OR Primary Neoplasms, Unknown OR Unknown Primary Neoplasm OR Unknown Primary Tumors OR Primary Tumor, Unknown OR Primary Tumors, Unknown OR Tumor, Unknown Primary OR Tumors, Unknown Primary OR Unknown Primary Tumor OR Neoplasm Metastasis, Unknown Primary OR Unknown Primary Neoplasm Metastasis

AND

Outcomes: Epidemiology OR Prevalence OR Prevalences OR Incidence OR Incidences

\section{Triagem e Seleção dos Estudos}

Um único revisor selecionou os trabalhos de forma independente, primeiro pelo título e resumo. Se o título englobava o tema e o resumo apresentava informações relevantes para os critérios de elegibilidade, o trabalho foi selecionado para uma leitura completa do texto. Em seguida, documentos de texto completo que preencheram os critérios de elegibilidade foram identificados e incluídos na revisão. O revisor pesquisou manualmente as listas de referências de todos os estudos selecionados para artigos adicionais relevantes que poderiam atender aos critérios de elegibilidade deste estudo. Os trabalhos que preencheram todos os critérios de seleção foram incluídos no estudo; os que não preencheram os critérios e/ou não se mostraram relevantes, foram excluídos. Os critérios de elegibilidade foram os seguintes:

- Artigos publicados na íntegra;

- Seres humanos;

- Case reports/classical articles sobre o tema;

-Consideramos tanto estudos prospectivos quanto retrospectivos;

- Tumores que acometem os maxilares que tenham sido provados histopatologicamente serem metástases de tumores primários em outras localizações.

- Artigos publicados em inglês, português e espanhol;

- Publicações entre 2000 e 2016. 


\section{Extração de Dados}

Quanto à questão focada, os dados foram extraídos dos trabalhos selecionados por um único revisor. Após uma avaliação preliminar dos trabalhos selecionados, os dados foram apresentados de forma descritiva em tabelas. As localizações dos tumores e o diagnóstico histopatológico dos relatos de casos estão apresentados na Tabela 1.
A Tabela 2 mostra os dados referentes aos achados clínicos e radiográficos, além do desfecho dos casos. As tabelas 3 e 4 mostram os mesmos dados, sendo referentes à amostra dos artigos de pesquisa, considerando os achados mais prevalentes de cada variável na amostra investigada.

Tabela 1. Localizações topográficas dos tumores e diagnósticos histopatológicos dos 33 casos relatados.

\begin{tabular}{|c|c|c|c|c|c|c|}
\hline AUTORES & SEXO & IDADE & $\begin{array}{c}\text { TUMOR } \\
\text { PRIMÁRIO }\end{array}$ & METÁSTASE & $\begin{array}{l}\text { DIAGNÓSTICO } \\
\text { HISTOLÓGICO }\end{array}$ & INTERVALO* \\
\hline \multirow[t]{8}{*}{ Bodner et al. } & $\mathrm{F}$ & 47 & Mama & Mandíbula (área posterior) & Carcinoma ductal & $\begin{array}{l}28.5 \text { meses } \\
\text { (média) }\end{array}$ \\
\hline & $\mathrm{F}$ & 67 & Pulmão & Mandíbula (área anterior) & $\begin{array}{l}\text { Carcinoma de células- } \\
\text { não-pequenas }\end{array}$ & \\
\hline & M & 67 & Reto & Maxila & Adenocarcinoma & \\
\hline & M & 71 & Pulmão & Mandíbula (área anterior) & $\begin{array}{l}\text { Carcinoma de células } \\
\text { não pequenas }\end{array}$ & \\
\hline & $\mathrm{F}$ & 44 & Mama & Mandíbula (área posterior) & Carcinoma ductal & \\
\hline & M & 65 & Tireoide & Mandíbula (área posterior) & $\begin{array}{c}\text { Carcinoma de células } \\
\text { de Hurtle }\end{array}$ & \\
\hline & $\mathrm{F}$ & 75 & Ureta & Mandíbula (área posterior) & Adenocarcinoma & \\
\hline & $\mathrm{F}$ & 80 & Parótida & Mandíbula (área posterior) & $\begin{array}{l}\text { Adenocarcnoma de } \\
\text { células basais }\end{array}$ & \\
\hline Uchiyama et al. & $\mathrm{F}$ & 73 & Estômago & Mandíbula (área anterior) & Adenocarcinoma & 10 meses \\
\hline Singh et al. & $\mathrm{F}$ & 42 & Colorretal & Mandíbula (área anterior) & Adenocarcinoma & 40 meses \\
\hline Cain, Goodlad, Denholm & $\mathrm{F}$ & 39 & Mama & $\begin{array}{l}\text { Glândulas salivares } \\
\text { submandibulares }\end{array}$ & Adenocarcinoma & 2 anos \\
\hline $\begin{array}{l}\text { Carrasco, Monasterio, } \\
\text { Ortega }\end{array}$ & M & 72 & Estômago & $\begin{array}{c}\text { Maxila e Mandíbula (área } \\
\text { anterior) }\end{array}$ & Carcinoma & $\begin{array}{l}\text { Primeiro } \\
\text { sinal }\end{array}$ \\
\hline Dumpala et al. & M & 24 & Costelas & Mandíbula (área posterior) & $\begin{array}{l}\text { Osteossarcoma } \\
\text { Condroblástico }\end{array}$ & 4 anos \\
\hline Jaffa et al. & $\mathrm{M}$ & 45 & Pâncreas & Mandíbula (área posterior) & Adenocarcinoma & $\begin{array}{l}\text { Primeiro } \\
\text { sinal }\end{array}$ \\
\hline Lutz et al. & M & 68 & íleo & Mandíbula (área posterior) & Tumor Estromal & $\begin{array}{l}\text { Primeiro } \\
\text { sinal }\end{array}$ \\
\hline Marchioni et al. & $\mathrm{F}$ & 78 & Mama & Maxila (Seios paranasais) & Carcinoma Ductal & 5 anos \\
\hline Menezes et al. & M & 54 & Próstata & Mandíbula (área posterior) & $\begin{array}{c}\text { Adenocarcinoma } \\
\text { acinar }\end{array}$ & 4 anos \\
\hline
\end{tabular}

INTERVALO*: Tempo entre o diagnóstico do tumor primário e o surgimento da metástase. NI†: Não informado.

\section{Análise dos Dados}

Os dados coletados a partir dos artigos de relatos/séries de casos e artigos de pesquisa foram analisados separadamente. Foi realizada uma análise de subgrupos, diferenciando os resultados dos relatos/séries de casos e pesquisas, considerando os seguintes dados: número total de indivíduos em cada subgrupo; sexo predominantemente acometido; idade mínima e máxima, mais média de idade; localizações topográficas dos tumores primários e secundários; número de indivíduos nos quais a lesão secundária foi o primeiro sinal de malignidade sistêmica; tipo histológico predominante. Destacamos a possibilidade de vieses de publicação, de idioma e de seleção dos estudos incluídos no trabalho, já que os estudos se limitaram quanto a período de publicação, a apenas três idiomas e a artigos com conteúdo publicados na íntegra, sendo este último critério responsável pela exclusão de 146 trabalhos. 
86 Tumores metastáticos para os maxilares

Tabela 2. Dados referentes aos achados clínicos, radiográficos e evolução dos 33 casos relatados

\begin{tabular}{|c|c|c|c|c|c|}
\hline AUTORES & SEXO & IDADE & SINAIS E SINTOMAS & ACHADOS IMAGINOLÓGICOS & EVOLUÇÃO \\
\hline \multirow[t]{8}{*}{ Bodner et al. } & $\mathrm{F}$ & 47 & Parestesia & $\mathrm{NI} *$ & Óbito entre 1 e 91 meses após \\
\hline & $\mathrm{F}$ & 67 & Inchaço & $\mathrm{NI} *$ & \\
\hline & M & 67 & Inchaço & $\mathrm{NI} *$ & \\
\hline & M & 71 & $\mathrm{NI} *$ & Lesão Radiolúcida Periapical & \\
\hline & $\mathrm{F}$ & 44 & Parestesia & $\mathrm{NI} *$ & \\
\hline & M & 65 & Inchaço & $\mathrm{NI} *$ & \\
\hline & $\mathrm{F}$ & 75 & Inchaço & $\mathrm{NI} *$ & \\
\hline & $\mathrm{F}$ & 80 & Inchaço & $\mathrm{NI} *$ & \\
\hline Uchiyama et al. & $\mathrm{F}$ & 73 & Inchaço/parestesia & Osteólise e Massa Tecidual & Óbito após 9 meses \\
\hline Singh et al & $\mathrm{F}$ & 42 & Inchaço & $\mathrm{NI*}$ & Óbito após 20 dias \\
\hline Cain, Goodlad, Denholm & $\mathrm{F}$ & 39 & Inchaço & $\mathrm{NI} *$ & $\mathrm{NI} *$ \\
\hline Carrasco, Monastério, Ortega & M & 72 & Inchaço eritematoso & Massa Tecidual & Óbito após 4 meses \\
\hline Dumpala et al. & M & 24 & Inchaço & Osteólise suave & Óbito após 1 mês \\
\hline Jaffa et al. & M & 45 & $\begin{array}{l}\text { Inchaço/dor/sangramento/ } \\
\text { parestesia }\end{array}$ & $\begin{array}{l}\text { Massa tecidual e Osteólise } \\
\text { suave }\end{array}$ & Óbito em alguns dias \\
\hline Lutz et al. & M & 68 & Inchaço/dor/parestesia & Osteólise e Massa Tecidual & Óbito após 11 meses \\
\hline Marchioni et al. & $\mathrm{F}$ & 78 & $\begin{array}{l}\text { Inchaço periorbital/diplopia/ } \\
\text { ptose palpebral }\end{array}$ & Osteólise e Massa Tecidual & Óbito após 4 meses \\
\hline Menezes et al. & M & 54 & $\begin{array}{l}\text { Abscesso/dor crônica/ } \\
\text { parestesia }\end{array}$ & Osteólise difusa & Óbito em menos de 1 ano \\
\hline Ogutcen-Toller, Metin, Yildiz & $\mathrm{F}$ & 50 & Inchaço/dor/parestesia & Osteólise & Óbito após 1 ano \\
\hline Misra et al.1 & $\mathrm{F}$ & 50 & Inchaço/dor & $\begin{array}{l}\text { Lesão destrutiva mista mal } \\
\text { definida }\end{array}$ & Óbito após 3 meses \\
\hline Pasupula et al. & $\mathrm{F}$ & 40 & Inchaço/dor & Osteólise destrutiva & $\mathrm{NI} *$ \\
\hline Rim et al. & $\mathrm{F}$ & 70 & Massa exofítica sangrante & $\mathrm{NI} *$ & $\mathrm{NI} *$ \\
\hline Sanchéz Jiménez et al. 4 & M & 63 & $\begin{array}{l}\text { Inchaço/ulceração gengival e } \\
\text { necrose }\end{array}$ & $\begin{array}{l}\text { Osteólise destrutiva da maxila, } \\
\text { apófise pterigoide e base de } \\
\text { órbita }\end{array}$ & Óbito após 2 semanas \\
\hline \multirow[t]{4}{*}{ Tamiolakis et al. 3} & $\mathrm{NI} *$ & 47 & Inchaço/dor & $\mathrm{NI} *$ & Sem retorno \\
\hline & $\mathrm{NI} *$ & $\mathrm{NI} *$ & Inchaço/ulcerações & $\mathrm{NI*}$ & Sem retorno \\
\hline & $\mathrm{NI} *$ & $\mathrm{NI} *$ & $\begin{array}{l}\text { In c h a ço / u I c e r a çã o / } \\
\text { sangramento/dor }\end{array}$ & $\mathrm{NI} *$ & $\mathrm{NI}^{*}$ \\
\hline & $\mathrm{F}$ & 69 & $\begin{array}{l}\text { Inchaço/obstrução nasal/ } \\
\text { epistaxe }\end{array}$ & $\mathrm{NI} *$ & Óbito após 1 mês \\
\hline Vahatalo, Ekfors, Syrjanen & M & 65 & Dor/parestesia & Osteólise & Óbito após 2 meses \\
\hline Agrawal et al. & M & 65 & Sinusite/cefaleia & $\begin{array}{l}\text { Massa tecidual da maxila ao } \\
\text { seio frontal }\end{array}$ & Óbito após 1 ano \\
\hline Çakmak et al. & M & 65 & Inchaço/dor & $\begin{array}{l}\text { Aumentado da absorção do } \\
\text { agente radiocontrastante na } \\
\text { região }\end{array}$ & $\begin{array}{l}\text { Acompanhamento de } 1 \text { ano } \\
\text { com quimioterapia }\end{array}$ \\
\hline Eichhorn et al. & $\mathrm{F}$ & 67 & Dor e parestesia & Osteólise & $\mathrm{NI} *$ \\
\hline Kattepur et al. & M & 70 & Obstrução nasal/epistaxe & $\mathrm{NI} *$ & Paciente recusou tratamento \\
\hline Mallikarjun et al. & $\mathrm{F}$ & 35 & Inchaço/parestesia & Osteólise irregular & $\mathrm{NI}{ }^{*}$ \\
\hline Soares et al. & M & 68 & Inchaço/dor/parestesia & Lesão mista lítica e esclerótica & Óbito após 3 semanas \\
\hline
\end{tabular}

NI*: Não informado. 
Tabela 3. Principais localizações topográficas dos tumores e diagnósticos histopatológicos nos 12 artigos de pesquisa

\begin{tabular}{|c|c|c|c|c|c|c|c|}
\hline AUTORES & $\mathbf{N}^{*}$ & SEXO† & IDADE‡ & TUMOR PRIMÁRIO & METÁSTASE & DIAGNÓSTICO HISTOLÓGICO & INTERVALO§ \\
\hline Kruse et al. & 51 & $28 \mathrm{~F}$ & 73.2 & Mama em $34 \%$ & $\begin{array}{r}\text { ATM (todos } \\
\text { casos) }\end{array}$ & Adenocarcinoma (72.97\%) & $\begin{array}{r}22 \text { - Primeiro } \\
\text { sinal }\end{array}$ \\
\hline Shen et al. & 19 & $11 \mathrm{M}$ & 47.9 & $\begin{array}{r}\text { Pulmão/Fígado em } \\
26.3 \%\end{array}$ & $\begin{array}{r}\text { Mandíbula } \\
(47.4 \%)\end{array}$ & $\begin{array}{l}\text { Carcinoma de células } \\
\text { escamosas/Carcinoma } \\
\text { hepatocelular }(26.3 \%)\end{array}$ & $\mathrm{NII}$ \\
\hline Daley e Darling & 38 & $22 \mathrm{M}$ & 69.6 & Próstata em 21\% & $\begin{array}{r}\text { Mandíbula } \\
\text { (53\%) }\end{array}$ & $\mathrm{NIII}$ & $\begin{array}{r}\text { 17-Primeiro } \\
\text { sinal }\end{array}$ \\
\hline D'Silva et al. & 114 & $52 \mathrm{M}$ & $\mathrm{NIII}$ & Mama em $25.4 \%$ & $\begin{array}{r}\text { Mandíbula } \\
(83.5 \%)\end{array}$ & $\mathrm{NIII}$ & $\begin{array}{r}\text { 66-Primeiro } \\
\text { sinal }\end{array}$ \\
\hline Fukuda et al. & 9 & $7 \mathrm{M}$ & 62.9 & Pulmão em 55.5\% & $\begin{array}{r}\text { Mandíbula } \\
(55.5 \%)\end{array}$ & Adenocarcinoma (44.4\%) & 3-Primeiro sinal \\
\hline Lim et al. & 41 & $26 \mathrm{M}$ & 55.2 & Fígado em $26.8 \%$ & $\begin{array}{r}\text { Mandíbula } \\
(65.8 \%)\end{array}$ & $\begin{array}{r}\text { Carcinoma hepatocelular } \\
(26.8 \%) \\
\text { Adenocarcinoma }(19.5 \%)\end{array}$ & $\begin{array}{r}\text { 11-Primeiro } \\
\text { sinal }\end{array}$ \\
\hline Seoane et al. & 39 & $21 \mathrm{M}$ & 62.3 & $\begin{array}{r}\text { Rins/pulmão/mama } \\
\text { em } 20.5 \%\end{array}$ & $\begin{array}{r}\text { Mandíbula } \\
(59 \%)\end{array}$ & Adenocarcinoma (53.8\%) & $\begin{array}{r}\text { Média de } 13.5 \\
\text { meses }\end{array}$ \\
\hline Antunes e Antunes & 10 & $6 \mathrm{~F}$ & 43 & $\begin{array}{r}\text { Tireoide/Próstata em } \\
30 \%\end{array}$ & $\begin{array}{r}\text { Mandíbula/ } \\
\text { Maxila (50\%) }\end{array}$ & Adenocarcinoma (50\%) & $\mathrm{NI} \mid \mathrm{I}$ \\
\hline McClure et al. & 26 & $16 \mathrm{M}$ & 64 & Pulmão em 34.6\% & $\begin{array}{r}\text { Mandíbula } \\
(69 \%)\end{array}$ & Adenocarcinoma (34.6\%) & $\begin{array}{r}\text { Média de } 31.5 \\
\text { meses }\end{array}$ \\
\hline Muttagi et al. & 19 & $11 \mathrm{~F}$ & 37.3 & $\begin{array}{r}\text { Mama/Tireoide em } \\
26.3 \%\end{array}$ & $\begin{array}{r}\text { Mandíbula } \\
(57.9 \%)\end{array}$ & Adenocarcinoma (36.8\%) & $\begin{array}{r}\text { Média de } 42.7 \\
\text { meses }\end{array}$ \\
\hline Shin et al. & 29 & $24 \mathrm{M}$ & 64.8 & $\begin{array}{r}\text { Pulmão/Fígado em } \\
34 \%\end{array}$ & $\begin{array}{r}\text { Gengiva sem } \\
\text { especificação } \\
(34 \%)\end{array}$ & $\begin{array}{r}\text { Carcinoma hepatocelular } \\
(34 \%)\end{array}$ & $\begin{array}{r}\text { Entre } 1 \text { e } 104 \\
\text { meses }\end{array}$ \\
\hline $\begin{array}{l}\text { van der Waal, Butter, } \\
\text { van der Waal }\end{array}$ & 24 & $12 \mathrm{~F} / \mathrm{M}$ & 60 & Mama em $25 \%$ & $\begin{array}{r}\text { Mandíbula } \\
(62.5 \%)\end{array}$ & Adenocarcinoma (62.5\%) & $\begin{array}{r}\text { Entre } 2 \text { e } 108 \\
\text { meses }\end{array}$ \\
\hline
\end{tabular}

$\mathrm{N}^{*}$ : Número total da amostra. SEXO†: Sexo mais acometido na amostra. IDADEł: Média de idade da amostra. INTERVALO§: Tempo entre o diagnóstico do tumor primário e o surgimento da lesão metastática. NI||: Não informado.

Tabela 4. Dados referentes aos principais achados clínicos, radiográficos e evoluções nos 12 artigos de pesquisa.

\begin{tabular}{|c|c|c|c|c|c|c|}
\hline AUTORES & $\mathbf{N}^{*}$ & SEXO+ & IDADEF & N/SINAIS E SINTOMAS§ & $\begin{array}{l}\text { N/ACHADOS } \\
\text { IMAGINOLÓGICOS|| }\end{array}$ & EVOLUÇÃO \\
\hline Kruse et al. & 51 & $28 \mathrm{~F}$ & 73.2 & 21-Dor/15-Inchaço/11-DTM & $\mathrm{N} \mid q$ & $\mathrm{~N} \mid q$ \\
\hline Shen et al. & 19 & $11 \mathrm{M}$ & 47.9 & 19-Inchaço/9-Dor/6-Parestesia & $\mathrm{N} \mid \boldsymbol{\eta}$ & 7 óbitos em 1 ano \\
\hline Daley e Darling & 38 & $22 \mathrm{M}$ & 69.6 & $\begin{array}{l}\text { 6-Parestesia/6-Alveolite/5- } \\
\text { Expansão óssea }\end{array}$ & 21-Osteólise & $\mathrm{N} \mid \boldsymbol{q}$ \\
\hline D'Silva et al. & 114 & $52 \mathrm{M}$ & $\mathrm{N} \mid \boldsymbol{q}$ & 35-Dor/19-parestesia/11-inchaço & Osteólise predominante & $\mathrm{N} \mid \boldsymbol{\eta}$ \\
\hline Fukuda et al. & 9 & $7 \mathrm{M}$ & 62.9 & 8-Inchaço/1-parestesia & 2-Osteólise/7- NIף & 8 óbitos em 1 ano \\
\hline Lim et al. & 41 & $26 \mathrm{M}$ & 55.2 & $\begin{array}{l}\text { Dor/Inchaço/Parestesia/ } \\
\text { Sangramento }\end{array}$ & Osteólise predominante & $\mathrm{N} \mid \boldsymbol{\eta}$ \\
\hline Seoane et al. & 39 & $21 \mathrm{M}$ & 62.3 & $\mathrm{~N} \mid$ ๆ & $\mathrm{N} \mid \boldsymbol{\eta}$ & $\begin{array}{l}\text { Sobrevida média de } 6 \\
\text { meses }\end{array}$ \\
\hline Antunes e Antunes & 10 & $6 \mathrm{~F}$ & 43 & $N \mid ף$ & $\mathrm{~N} \mid q$ & $\mathrm{~N} \mid q$ \\
\hline McClure et al. & 26 & $16 \mathrm{M}$ & 64 & 5-Parestesia/3-fratura patológica & Osteólise predominante & 19 óbitos em 4 meses \\
\hline Muttagi et al. & 19 & $11 \mathrm{~F}$ & 37.3 & Inchaço/Dor/assimetria/parestesia & $\mathrm{N} \mid \boldsymbol{q}$ & 10 óbitos em 6 meses \\
\hline Shin et al. & 29 & $24 \mathrm{M}$ & 64.8 & 21-Inchaço/5-dor/3-sangramento & $N \mid ף$ & 25 óbitos em 1 ano \\
\hline $\begin{array}{l}\text { van der Waal, Butter, van } \\
\text { der Waal }\end{array}$ & 24 & $12 \mathrm{~F} / \mathrm{M}$ & 60 & Dor/Inchaço/Parestesia & Osteólise predominante & $\begin{array}{l}\text { Sobrevida média de } 6 \\
\text { meses }\end{array}$ \\
\hline
\end{tabular}




\section{RESULTADOS}

\section{Resultados da busca e seleção}

Foram escolhidos 35 trabalhos relevantes para a extração de dados, os quais foram selecionados após a leitura completa e a constatação de que cumpriram todos os critérios de inclusão. Sendo eles, 12 artigos de pesquisa e 23 artigos do tipo relato/ série de casos, que relatavam 33 casos.

\section{Características da amostra}

Foi encontrado um total de 33 casos relatados de indivíduos acometidos por metástases nos maxilares, dos quais a maioria 16 (48.5\%), eram do sexo feminino, já que três indivíduos em uma das séries de casos não tinham os sexos relatados. Os dados coletados a partir dos relatos de casos revelaram que a idade dos pacientes variou dos 24 a 80 anos com média de 59.19 .

\section{Localizações topográficas e tipos histológicos}

Entre os locais de acometimento das lesões metastáticas, destacou-se a mandíbula com 26 (79\%) dos casos, considerando tanto metástases em tecidos moles quanto duros. Já em relação às localizações mais comuns para os tumores primários, o local de maior acometimento foi a mama com 7 (21.2\%) casos, seguida pela tireoide e fígado com $4(12.1 \%)$ casos cada. Em $8(24.2 \%)$ casos, os tumores primários eram desconhecidos ao diagnóstico das lesões orais metastáticas. Nos demais casos, com histórico prévio de malignidade, o tempo entre o diagnóstico do tumor primário e da lesão metastática variou entre 4 meses a até 7 anos. Com relação aos tipos histológicos dos tumores, o adenocarcinoma foi o principal padrão, sendo encontrado em 17 (51.1\%) dos casos. As demais localizações dos tumores primários, secundários e tipos histológicos dos relatos/séries de casos estão exibidos na Tabela 1.

\section{Características da amostra}

Foi encontrado um total de 419 indivíduos acometidos por metástases nos maxilares, dos quais 222 (53\%) eram do sexo masculino. Os dados coletados a partir da revisão dos artigos de pesquisa revelaram que a idade dos pacientes variou dos 4 meses a 90 anos com uma média de 58.20.

\section{Localizações topográficas e tipos histológicos}

Entre os locais de acometimento das lesões metastáticas, destacou-se a mandíbula com 232 (55.3\%) dos casos, considerando tanto metástases em tecidos moles quanto duros. Já em relação às localizações mais comuns para os tumores primários, foram o pulmão e mama, ambos com 83 (19.8\%) casos cada. Em 168 (40.1\%) casos, os tumores primários eram desconhecidos ao diagnóstico das lesões orais metastáticas. Nos demais casos, com histórico prévio de malignidade, o tempo entre o diagnóstico do tumor primário e da lesão metastática variou entre 1 mês a até 108 meses. Com relação aos tipos histológicos dos tumores, o adenocarcinoma foi o principal padrão sendo encontrado em 120 (28.7\%) dos casos, uma vez que este dado não foi relatado em 152 (36.3\%) casos. As demais localizações dos tumores primários, secundários e tipos histológicos dos artigos de pesquisa estão exibidos na Tabela 3.

\section{DISCUSSÃO}

Os tumores metastáticos que comprometem o sistema estomatognático representam cerca de $1 \%$ de todas as malignidades orais, apresentando-se tanto em tecidos moles quanto nos ossos maxilares, constituindo um desafio diagnóstico ${ }^{6}$. Relata-se que cerca de $30 \%$ dos casos de tumores metastáticos dos maxilares (TMM) representam o primeiro sinal de disseminação de um tumor primário de origem desconhecida ${ }^{1-5}$. Porém, nos estudos de McClure et al ${ }^{7}$. e $D^{\prime}$ silva et al. $^{8}$, os autores encontraram uma frequência muito maior, em $62 \%$ e $57.8 \%$ da amostra, respectivamente; a metástase oral foi o primeiro sinal de malignidade.

As células neoplásicas disseminam-se pelos vasos sanguíneos e linfáticos, circulam no sistema cardiovascular, sobrevivem aos diversos mecanismos de defesa do organismo e se estabelecem na microcirculação do tecido alvo extravasando-se através das paredes endoteliais, ganhando um novo ambiente, onde estabelecem um tumor metastático. O sucesso das células metastáticas em constituir o tumor secundário depende de sua capacidade de manter-se no novo ambiente, principalmente por meio da angiogênese. Usualmente, a metástase nos maxilares ocorre através de embolização por via sanguínea, pois os ossos maxilares são quase que desprovidos de vasos linfáticos. Essa disseminação requer a presença de medula óssea hematopoeticamente ativa ${ }^{5,9}$.

Discorrendo acerca do desfecho primário do presente estudo sobre as localizações dos tumores, a região posterior da mandíbula é a mais afetada, principalmente em pacientes dentados, já que a presença de dentes nos ossos maxilares parece ser um determinante importante no local preferencial para a metástase, uma vez que essa condição parece estar associada à ocorrência de locais hematopoeticamente ativos que permitiriam a atração de células tumorais ${ }^{5,9,10,11}$. Mesmo em pacientes edêntulos, com focos de defeitos osteoporóticos, podem existir os locais antes citados, permitindo também a atração celular, sendo que nestes pacientes as lesões metastáticas são igualmente distribuídas entre a língua e a mucosa alveolar ${ }^{5}$. A inflamação desempenha um papel importante na atração das células neoplásicas metastáticas para a gengiva inserida. Tais células podem ficar aprisionadas na rede capilar de uma gengiva inflamada, constituindo este um microambiente ideal para a progressão tumoral. Essa informação sustenta-se no fato de que a inflamação crônica tem sido associada a várias etapas da tumorigênese, desde a transformação celular, promoção, sobrevivência, proliferação, invasão, angiogênese e metástase ${ }^{5,9}$.

É sugerido que as metástases para cabeça e pescoço usualmente 
associam-se a neoplasias pulmonares. Se não há sinais de doença pulmonar, é possível que as células neoplásicas tenhamse disseminado silenciosamente através do plexo venoso de Batson ou através do ducto torácico. O plexo venoso de Batson estende-se desde o crânio até o sacro. Este plexo teoricamente oferece menor resistência à propagação de êmbolos tumorais, permitindo o fluxo retrógrado, ignorando filtros pulmonares $s^{5,9,10}$.

Os TMM podem ser descobertos após uma exodontia, sugerindo que a neoplasia estava presente no local antes do procedimento e que por falta de um exame clínico adequado, ou por diagnóstico errôneo, o clínico foi levado a realizar a extração do dente, inclusive favorecendo a progressão tumoral com o reparo tecidual como acelerador. No entanto, algumas metástases podem desenvolver-se só após a exodontia, aproveitando a angiogênese que acompanha o processo de reparo; nesses casos a exodontia poderia funcionar como um promotor de atração das células neoplásicas ${ }^{5,9,10}$. Cinco critérios são considerados para diagnosticar uma lesão como metastática: (1). Deve ser identificado o tumor primário, (2) A lesão secundária deve ser histologicamente igual à primária, (3) A possibilidade de extensão direta da neoplasia primária deve ser excluída, (4) $\mathrm{O}$ aspecto histopatológico é distinto do de uma malignidade típica de origem oral, (5). Há evidências de um tumor primário mal controlado.

Quanto às diferentes populações mundiais, alguns estudos ao redor do mundo mostram certas particularidades. No Japão, a maior ocorrência de câncer de pulmão, tireoide, fígado, esôfago e estômago determinam a maior frequência de lesões metastáticas nessa população do que quando comparada a dos $E A^{13}$. Shin et $\mathrm{al}^{14}$, em seu estudo realizado com 29 pacientes em um centro médico coreano, encontraram o fígado, o pulmão e o estômago como as principais localizações primárias, já Lim et al. ${ }^{15}$ em um estudo com 41 pacientes também na Coreia, encontraram como sendo o fígado, pulmão e tireoide, respectivamente, sendo a tireoide e não a mama a origem mais

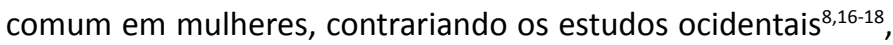
levando-nos a supor a possibilidade de uma tendência regional na origem das lesões metastáticas.

Considerando a idade, há uma maior frequência de TMM em pacientes entre as 5ㅇ e 8 o décadas de vida ${ }^{1-18}$. Já se tratando de gênero, a proporção de neoplasias metastáticas para o sistema estomatognático parece ser igual para ambos os sexos. No entanto, considerando alguns sítios acometidos, existem sutis diferenças, sendo relatado que para os tumores cujas metástases comprometem os ossos maxilares a proporção homem-mulher é de 1:1.1 e naqueles que metastatizam para tecidos moles essa proporção é de $\mathrm{e}^{2: 15,10}$.

Acerca dos desfechos secundários, as características clínicas e radiográficas dos TMM's, em estágios iniciais de desenvolvimento podem assemelhar-se a lesões hiperplásicas ou reacionais. As lesões gengivais usualmente exibem aspecto polipoide ou exofítico, bem vascularizadas, portanto, de coloração avermelhada ou azulada, podendo sangrar ${ }^{19}$. A apresentação clínica dos TMM é altamente variável, o que representa possibilidades de erros diagnósticos. Podem fazer diagnóstico diferencial com o granuloma piogênico20, fibroma oral/fibromatose, abscesso periodontal, granuloma periférico de células gigantes, neuralgia trigeminal ${ }^{21}$ e carcinoma de células escamosas, carcinoma primário intraósseo, tumores odontogênicos malignos, obstruções nasais e sinusites ${ }^{22,23}$, tumores malignos nasais primários ${ }^{24}$, tumores malignos de glândulas salivares intraósseos, sarcomas, lesões periapicais/ císticas ${ }^{25}$, tumores odontogênicos, osteomielites, e até casos de doença periodontal ${ }^{26}$, a depender do aspecto e localização da lesão oral.

Entre os diferentes sinais e sintomas apresentados pelos relatos de tumores metastáticos para os maxilares, a literatura tem chamado atenção para um sintoma que tem se apresentado de forma bastante frequente nas metástases intraósseas em mandíbula que é a "síndrome do queixo dormente" (numb chin syndrome), caracterizada por hiperestesia, parestesia, ou dor no queixo na região inervada pelo ramo do nervo alveolar inferior. Apesar de não ser um sintoma exclusivo, deve ser visto como um sintoma alarmante em pacientes com histórico de doença maligna e/ou em idosos 2,19,27. $^{2}$.

Não existe aspecto radiográfico patognomônico para os TMM. Suas características podem variar de inicial ausência de alguma manifestação, osteólise, à presença de radiopacidade mal definida. O fenótipo das lesões ósseas metastáticas resulta do equilíbrio entre a atividade osteoblástica e a osteoclástica. As observações epidemiológicas destacam que os TMM provenientes da próstata, representam lesões osteoblásticas, enquanto que, TMM com origens no rim, pulmão ou mama geralmente são lesões osteolíticas ${ }^{5,9,28}$.

Considerando estudos com enfoque nas localizações topográficas dos tumores, Seoane et al. ${ }^{17}$ encontraram em uma amostra de 39 pacientes, $28.2 \%$ de lesões localizadas em tecidos moles. Destes casos em tecidos moles, $63.6 \%$ eram localizados na gengiva, sendo $85.7 \%$ localizados na maxila. Por outro lado, a mandíbula foi a principal localização para tumores intraósseos, acometendo $77.8 \%$ desses casos. No estudo de Shin et al $^{14}$ são encontrados achados extremamente contrastantes com a literatura. Os autores encontraram em uma amostra de 29 pacientes, apenas 4 casos de metástases intraósseas, sendo os outros 25 casos todos em tecidos moles, sobretudo a gengiva, achado que os autores não souberam explicar.

Considerando a ocorrência de casos atípicos entre a amostra dos artigos, podemos ressaltar o caso relatado por Cain, Goodlad, Denholm ${ }^{29}$ de uma paciente do sexo feminino de apenas 39 anos, com carcinoma de mama e que veio a desenvolver uma lesão metastática para a glândula submandibular direita após dois anos de acompanhamento, sendo tratada por ressecção cirúrgica da glândula. Após mais de um ano a glândula submandibular esquerda também foi acometida, sendo o único caso de metástases bilaterais metacrônicas para as glândulas submandibulares encontrado na literatura consultada. Um outro caso é o relatado por Vähätalo, Ekfors, Syrjänen ${ }^{30}$ de um paciente masculino, de 65 anos, sem história de maliginidade, apresentando uma lesão osteolítica no ângulo mandibular 
direito associada a parestesia labial, sendo diagnosticado com adenocarcinoma metastático cuja origem foi rastreada para o pâncreas. A raridade do caso está no fato de que na mesma localização da lesão mandibular, 10 anos antes, um odontogênico ceratocístico havia sido removido. Porém, não há evidências de que o odontogênico ceratocístico tenha tido alguma relação com o local da metástase.

Como segundo desfecho primário do estudo, demos enfoque à histopatologia das lesões metastáticas. A maioria dos casos era de tumores de origem epitelial, sendo o adenocarcinoma o tipo mais frequente, estabelecendo uma afinidade entre malignidades de origem epitelial e os maxilares, necessitando ainda de estudos específicos5. Entre os artigos de relatos de casos houve apenas dois casos atípicos que envolveram um osteosarcoma condroblástico ${ }^{31}$ e um tumor de tipo estromal ${ }^{32}$.

Para o tratamento e prognóstico dos TMM são considerados o sítio de origem do tumor primário e o grau de disseminação metastática. Se o tumor primário é descoberto e tratado com sucesso, a metástase oral igualmente o será. Se o tumor primário é recorrente ou existem metástases disseminadas, a lesão nos maxilares pode ser tratada de forma conservadora, no intuito de otimizar a qualidade de vida ao máximo. O tratamento dos TMM pode envolver ressecção, radiação, quimioterapia, tratamento de suporte ou a combinação de modalidades ${ }^{5,17}$, ${ }^{18,33}$. Infelizmente, indivíduos com metástases orais representam um grupo de pacientes em estado terminal com pouco tempo de sobrevida, muitas vezes vindo a sucumbir antes de terem suas lesões diagnosticadas, o que torna desconhecida a verdadeira incidência das lesões metastáticas que acometem os maxilares ${ }^{34,35}$.
Com base em nossa análise da literatura podemos concluir que os seguintes passos devem ser estritamente seguidos na avaliação de lesões suspeitas: (1) Revisar exaustivamente a história clínica do paciente; (2) Analisar os exames imaginológicos; (3) Pesquisar a existência de história prévia de neoplasia; para tal fim, devemse investigar todas as informações existentes sobre ela; (4) Realizar biópsia da lesão oral; (5) Analisar histopatologicamente a lesão e avaliar a necessidade de técnicas auxiliares ao diagnóstico histopatológico de rotina (histoquímica, imunohistoquímica, microscopia eletrônica).

Com relação às dificuldades do presente estudo, além dos possíveis vieses já relatados na metodologia, há o número limitado de artigos incluídos devido ao fato de considerarmos como critério de inclusão a necessidade de incluir artigos disponíveis na íntegra, já que um grande número de trabalhos não eram disponibilizados de forma integral o que impossibitava a coleta de dados importantes para a revisão.

Em conclusão, os tumores metastáticos dos maxilares são lesões que não possuem características clínicas ou imaginológicas que caracterizam essas lesões, se apresentando com sinais e sintomas similares a alterações benignas, o que está relacionado a um diagnóstico desafiador e tardio com prognóstico sombrio de curta sobrevida. Acometem mais comumente a região mandibular posterior entre a quinta e oitava décadas de vida, em ambos os sexos. O fato de, em muitos casos, ser o primeiro sinal da existência de malignidade destaca o papel preponderante do cirurgião-dentista no diagnóstico precoce dessas lesões. Sugere-se um cuidadoso exame clínico bem como uma abordagem multidisciplinar.

\section{REFERÊNCIAS}

1. Misra SR, Shankar YU, Rastogi V, Maragathavalli G. Metastatic hepatocellular carcinoma in the maxilla and mandible, an extremely rare presentation. Contemp Clin Dent. 2015 Mar; 6(Suppl 1):S117-21. doi: 10.4103/0976237X.152966 PubMed PMID: 25821363.

2. Antunes AA, Antunes AP. Gnathic bone metastasis: a retrospective study of 10 cases. Braz J Otorhinolaryngol. 2008 Jul-Aug; 74(4):561-5. PubMed PMID: 18852982.

3. Tamiolakis D, Tsamis I, Thomaidis V, Lambropoulou M, Alexiadis G, Venizelos I, et al. Jaw bones metastases: four cases. Acta Dermatovenerol Alp Pannonica Adriat. 2007 Mar;16(1):21-5. PubMed PMID: 17992450.

4. Sánchez-Jiménez J, Acebal-Blanco F, Arévalo-Arévalo RE, Molina-Martinez M. Metastatic tumours in upper maxillary bone of esophageal adenocarcinoma. A case report. Med Oral Patol Oral Cir Bucal. 2005 May-Jul; 10(3):252-7. PubMed PMID: 15876970.

5. Muttagi SS, Chaturvedi P, D'Cruz A, Kane S, Chaukar D,Pai P. Metastatic tumors to the jaws bonés: retrospective analysis from an Indian tertiary referral center. Indian J Cancer. 2011 Apr-Jun; 48(2):234-9. doi: 10.4103/0019-509X.82894. PubMed PMID: 21768673.

6. Singh T, Amirtham U, Satheesh CT, Lakshmaiah KC, Suresh TM, Babu KG et al. Floor-of-mouth metastasis in colorectal cancer. Ann Saudi Med. 2011 Jan-Feb; 31(1):87-9. doi: 10.4103/0256-4947.70583. PubMed PMID: 20864784.

7. McClure SA, Movahed R, Salama A, Ord RA. Maxillofacial metastases: a retrospective review of one institution's 15-year experience. J Oral Maxillofac Surg. 2013 Jan;71(1): 178-88. doi: 10.1016/j.joms.2012.04.009. PubMed PMID: 22705221.

8. D'Silva NJ, Summerlin DJ, Cordell KG, Abdelsayed RA, Tomich CE, Hanks CT, et al. Metastatic tumors in the jaws: a retrospective study of 114 cases. J Am Dent Assoc. 2006 Dec;137(12):1667-72. PubMed PMID: 17138711.

9. Mallikarjun JH, Mujib BR, Naik R, Patil ST. Metastatic small cell carcinoma of the cervix to the oral cavity: a rare case report and an insight into pathogenesis of metastasis. J Oral Maxillofac Pathol. 2015 May-Aug; 19(2):247-50. doi: 10.4103/0973-029X.164541. PubMed PMID: 26604504.

10. Daley T, Darling MR. Metastases to the Mouth and Jaws: a Contemporary Canadian Experience. J Can Dent Assoc. 2011; 77:b67. PubMed PMID: 21683026.

11. Çakmak Ö, Tarhan H, Küçük Ü, Ilbey YO. Prostatic adenocarcinoma with initial metastatic spread to the mandible. Turk J Urol. 2015 Sep; 41(3):149-51. doi: 10.5152/tud.2015.36043. PubMed PMID: 26516599.

12. Carrasco EC, Monasterio RB, Ortega A. Presentación de doble metástasis en territorio máxilo facial: reporte de caso clínico. Acta odontol. Venez. [internet]. 2012[acesso 2017 Jan 10]; 50(1). Disponível em: http://www.actaodontologica. com/ediciones/2012/1/art-15/.

13. Fukuda M, Miyata M, Okabe K, Sakashita H. A case series of 9 tumors metastatic to the oral and maxillofacial region. J Oral Maxillofac Surg. 2002 Aug; 60(8):942-4. PubMed PMID: 12149744. 
14. Shin SJ, Jong-Lyel R, Choi SH, Nam SY, Kim SY, Kim SB, et al. Metastatic carcinomas to the oral cavity and oropharynx. Korean J Pathol. 2012 Jun 46(3):266-71. doi: 10.4132/KoreanJPathol.2012.46.3.266. PubMed PMID: 23110013.

15. Lim SY, Kim SA, Ahn SG, Kim HK, Kim SG, Hwang HK. Metastatic tumours to the jaws and oral soft tissues: a retrospective analysis of 41 Korean patients. Int J Oral Maxillofac Surg. 2006 May;35(5):412-5. doi: 10.1016/j.ijom.2005.12.001. PubMed PMID: 16473498.

16. Kruse AL, Luebbers HT, Obwegeser JA, Edelmann L, Graetz KW. Temporomandibular disorders associated with metastases to the temporomandibular joint: a review of the literature and 3 additional cases. Oral Surg Oral Med Oral Pathol Oral Radiol Endod. 2010 Aug; 110(2):e21-8. doi: 10.1016/j.tripleo.2010.02.031. PubMed PMID: 20659692.

17. Seoane J, Van der Waal I, Van der Waal RI, Cameselle-Teijeiro J, Antón I, Tardio A, et al. Metastatic tumours to the oral cavity: a survival study with a special focus on gingival metastases. J Clin Periodontol. 2009 Jun;36(6):488-92. doi: 10.1111/j.1600-051X.2009.01407.x. PubMed PMID: 19508248.

18. van der Waal RI, Buter J, van der Waal I. Oral metastases: report of 24 cases. Br J Oral Maxillofac Surg. 2003 Feb;41(1):3-6. PubMed PMID: 12576032.

19. Bodner L, Sion-Vardy N, Geffen DB, Nash M. Metastatic tumors to the jaws: A report of eight new cases. Med Oral Patol Oral Cir Bucal. 2006 Mar 1;11(2):E132-5. PubMed PMID: 16505790

20. Rim JH, Moon SE, Chang MS, Kim JA. Metastatic hepatocellular carcinoma of gingiva mimicking pyogenic granuloma. J Am Acad Dermatol. 2003 Aug; 49(2):342-3. PubMed PMID: 12894096.

21. Menezes JDS, Cappellari PFM, Capelari MM, Gonçalves PZ, Toledo GL, Toledo JL Filho, et al. Mandibular metastasis of adenocarcinoma from prostate cancer: case report according to epidemiology and current therapeutical trends of the advanced prostate cancer. J Appl Oral Sci. 2013 Sep-Oct; 21(5):490-5. doi: 10.1590/1679-775720130148.

22. Agrawal S, Jayant K, Agarwal RK, Dayama KG, Arora S. An unusual case of metastatic male breast cancer to the nasopharynx-review of literature. Ann Palliat Med. 2015 Oct; 4(4):233-8. doi: 10.3978/j.issn.2224-5820.2015.08.02. PubMed PMID: 26541404.

23. Kattepur AK, Patil DB, Krishnamoorthy N, Srinivas KG, Swa,y S, Amaendra S, et al. Isolated nasopharyngeal metastasis from hepatocellular carcinoma. Int J Surg Case Rep. 2014;5(3):115-7. doi: 10.1016/j.ijscr.2013.12.009. PubMed PMID: 24509427.

24. Marchioni D, Monzani D, Rossi G, Rivasi F, Presutti L. Breast carcinoma metastases in paranasal sinuses, a rare occurrence mimicking a primary nasal malignancy: case report. Acta Otorhinolaryngol Ital. 2004 Apr;24(2):87-91. PubMed PMID: 15468998.
25. Eichhorn W, Wehrmann M, Blessmann M, Pohlenz P, Blake F, Schmelzle R, et al. Metastases in odontogenic cysts: literature review and case presentation. Oral Surg Oral Med Oral Pathol Oral Radiol Endod. 2010 Apr;109(4):582-6. PubMed PMID: 20303056.

26. Ogütcen-Toller M, Metin M, Yildiz L. Metastatic breast carcinoma mimicking periodontal disease on radiographs. J Clin Periodontol. 2002 Mar;29(3):269-71. PubMed PMID: 11940148

27. Soares EC, Cosa FW, Rocha-Filho FD, Ferreira FV, Alves AP. Metastatic prostate adenocarcinoma associated with numb chin syndrome. J Craniofac Surg. 2011 Nov;22(6):2366-8. doi: 10.1097/SCS.0b013e318231e5a1. PubMed PMID: 22134281.

28. Uchiyama $\mathrm{Y}$, Murakami S, Kakimoto N, Nakatani A, Kishino M, Hamab $\mathrm{Y}$, et al. Diagnostic imaging findings for mandibular metastasis from gastric adenocarcinoma. Oral Surg Oral Med Oral Pathol Oral Radiol Endod. 2009 Jun;107(6):e49-53. doi: 10.1016/j.tripleo.2009.03.004.. PubMed PMID: 19464643

29. Cain AJ, Goodlad J, Denholm SW. Metachronous bilateral submandibular gland metastases from carcinoma of the breast. J Laryngol Otol. 2001 Aug;115(8):683-4. PubMed PMID: 11535161.

30. Vähätalo K, Ekfors T, Syrjänen S. Adenocarcinoma of the pancreas metastatic to the mandible. J Oral Maxillofac Surg. 2000 Jan; 58(1):110-4. PubMed PMID: 10632176. doi: http://dx.doi.org/10.1016/S0278-2391(00)80028-2.

31. Dumpala RK, Guttikonda VR, Yeluri S, Madala J. Oral metastasis of chondroblastic osteosarcoma. Contemp Clin Dent. 2012 Jul; 3(3):367-9. doi: 10.4103/0976-237X.103640 PubMed PMID: 23293503.

32. Lutz JC, El-Bouihi M, Vidal N, Fricain JC, Rober $M$, Deminière $C$, et al. Mandibular metastases from an ileum stromal tumor. Rev Stomatol Chir Maxillofac. 2008 Dec;109(6):399-402. doi: 10.1016/j.stomax.2008.09.010. PubMed PMID: 19010506.

33. Shen $M L$, Kang J, Wen YL, Ying WM, Hua CG, Tang XF, et al. Metastatic tumors to the oral and maxillofacial region: a retrospective study of 19 cases in West China and review of the Chinese and English literature. J Oral Maxillofac Surg. 2009 Apr;67(4):718-37. doi: 10.1016/j.joms.2008.06.032. PubMed PMID: 19304027.

34. Jaffa NR, Adam D, Akhtar S, Kyzas PA. Pancreatic adenocarcinoma presenting as mandibular tumor: case report. Oral Surg Oral Med Oral Pathol Oral Radiol. 2014 Jan;117(1):23-6. PubMed PMID: 24184062.

35. Pasupula AP, Dorankula SPR, Trokala MR, Kumar MP. Metastatic follicular thyroid carcinoma to the mandible. Indian J Dent Res. 2012 Nov-Dec;23(6):843. doi: 10.4103/0970-9290.111292. PubMed PMID: 23649086. 